\title{
Efficient Light Scattering through Thin Semi-Transparent Objects
}

\author{
Jeppe Revall Frisvad* \\ Niels Jørgen Christensen ${ }^{\dagger}$ \\ Informatics and Mathematical Modelling \\ Technical University of Denmark
}

Peter Falster ${ }^{\ddagger}$

\begin{abstract}
This paper concerns real-time rendering of thin semi-transparent objects. An object in this category could be a piece of cloth, eg. a curtain. Semi-transparent objects are visualized most correctly using volume rendering techniques. In general such techniques are, however, intractable for real-time applications. Surface rendering is more efficient, but also inadequate since semi-transparent objects should have a different appearance depending on whether they are front-lit or back-lit. The back-lit side of a curtain, for example, often seems quite transparent while the front-lit side seems brighter and almost opaque. To capture such visual effects in the standard rendering pipeline, Blinn [1982] proposed an efficient local illumination model based on radiative transfer theory. He assumed media of low density, hence, his equations can render media such as clouds, smoke, and dusty surfaces. Our observation is that Chandrasekhar [1960] has derived the same equations from a different set of assumptions. This alternative derivation makes the theory useful for realistic real-time rendering of dense, but thin, semitransparent objects such as cloth. We demonstrate that application of the theory in this new area gives far better results than what is obtainable with a traditional real-time rendering scheme using a constant factor for alpha blending.
\end{abstract}

CR Categories: I.3.3 [Computer Graphics]: Picture/Image Generation I.3.7 [Computer Graphics]: Three-Dimensional Graphics and Realism

Keywords: cloth rendering, global illumination, optically thin media, real-time rendering, semi-transparent surfaces

\section{Introduction}

Cloth is a physically complex phenomenon which we surround ourselves with in everyday life. An important aspect of realistic image synthesis is, therefore, the modeling and rendering of clothes and other objects composed of fabrics.

Fabrics are often modeled as infinitely thin surfaces rather than volumes. This representation makes it difficult to account for scattering of light through the material. The result is that the rendering must often be contempt with evaluation of a BRDF (Bidirectional Reflectance Distribution Function) capturing the reflection off the surface. Sometimes transmission through the surface is approximated by use of an alpha blending factor depending on the weave pattern of the cloth. Dependence on the surrounding illumination is, however, not included in this blending factor, why surface models

\footnotetext{
*e-mail: jrf@imm.dtu.dk

†e-mail: njc@imm.dtu.dk

‡e-mail: pfa@imm.dtu.dk
}

(C)ACM, 2005. This is the author's version of the work. It is posted here by permission of ACM for your personal use. Not for redistribution. The definitive version was published in Proceedings of GRAPHITE 2005, pp. 135-138. in general are unable to capture the different appearance that fabric has when it is front-lit as opposed to when it is back-lit.

Semi-transparent fabrics represented by surfaces should be thought of as optically thin media with respect to light scattering. Optical thickness of a medium depends not only on the spatial thickness, but also on the scattering properties of the material. Consider a piece of cloth represented only by its surface, it is reasonable to assume that such a medium is locally plane-parallel. Light transfer for plane-parallel problems has been studied extensively in the field of radiative transfer. The first definitive text of that field was provided by Chandrasekhar [1960]. To find an efficient method for cloth rendering capturing the visual effects mentioned before, we will draw on the theory presented in that text.

Blinn was the first, in his seminal paper [1982], to employ radiative transfer theory for visualization of semi-transparent media, also referred to as participating media. Subsequently much has been done to expand the idea of volume rendering using radiative transfer theory, see eg. [Kajiya and Von Herzen 1984; Rushmeier and Torrance 1987; Max 1995]. Blinn's approach has been regarded as useful for scattering off and through cloud layers and dusty surfaces only, since his equations were derived under the assumption that the rendered medium has a low density. Our observation is that the same equations were derived in [Chandrasekhar 1960] under a different assumption, namely that the rendered medium is optically thin. This indicates that the equations are also useful for rendering of dense, but optically thin, semi-transparent objects such as clothes. If that is the case, we have an efficient local illumination model able to capture visual effects resulting from transmission and isotropic scattering of light, with a new area of application. In the following we will demonstrate that the equations are, indeed, useful for cloth rendering.

\section{Related Work}

Models for cloth rendering can be divided into two categories: Surface models and volume models. Usually the surface models do not account for light transmitted through the surface except perhaps for the alpha blending factor mentioned before, see [Adabala et al. 2003]. As a compensation the simple surface models are fast.

Adabala [2004] provides an overview of the different approaches to cloth rendering and describes the volume models under the flag of three-dimensional textures for representation of micro geometry. This alone indicates that few existing models deliberately deal with the visual effects addressed in this paper. Volume models, such as the one presented in [Messnier et al. 2000], the one in [Daubert and Seidel 2002], or the lumislice model presented eg. in [Chen et al. 2003], should be able to capture the effect of light transmitted through textile, but they are costly to evaluate. Hence, we opt for a model which renders surface representations of fabric while still being able to capture that thin fabric changes appearance depending on whether it is front-lit or back-lit.

Daubert et al. [2001] do pre-computation of complex viewdependent BRDFs based on weaving or knitting patterns and obtain interactive frame rates. Their BRDFs also include view-dependent transparency based on holes in the considered pattern, but, since BRDFs by definition do not capture transmitted light, their model 
does not capture the different appearance of front-lit and back-lit cloth.

One approach which does take transmitted light into account was presented in [Volevich et al. 1997]. Here a BSDF (Bidirectional Scattering Distribution Function) is evaluated using Monte Carlo ray tracing of micro-elements representing fibers in the cloth. Hence, this is a surface based model dealing with transmission, but it does not have the advantage of being fast and is solely intended for off-line rendering.

\section{Backgound Theory}

When Blinn [1982] derived his equations for visualization of clouds and dusty surfaces, he did not start from the volume rendering equation. He used a different geometrical model based on a sparsely populated cloud of particles. The volume rendering equation is based on [Chandrasekhar 1960] and emerged later in [Kajiya and Von Herzen 1984].

To understand how Blinn's equations can be derived under a different set of assumptions, we need to review some of the theory on plane-parallel problems presented in [Chandrasekhar 1960]. Along the way, we will argue why the assumptions made are reasonable with respect to cloth rendering.

The following is a version of the volume rendering equation for scattering media ${ }^{1}$ expressed in terms of thickness $s$ defined along a ray traversing the medium in a certain direction $\boldsymbol{\omega}$ :

$$
\begin{aligned}
L(s) & =L(0) e^{-\tau(s, 0)} \\
& +\int_{0}^{s} e^{-\tau\left(s, s^{\prime}\right)} \sigma_{t}\left(s^{\prime}\right) \int_{\Omega_{4 \pi}} p\left(s^{\prime}, \omega^{\prime}\right) L\left(s^{\prime}, \omega^{\prime}\right) d \omega^{\prime} d s^{\prime},
\end{aligned}
$$

where $\tau\left(s, s^{\prime}\right)$ is the optical thickness of the material between the points $s$ and $s^{\prime}$ given as

$$
\tau\left(s, s^{\prime}\right)=\int_{s^{\prime}}^{s} \sigma_{t}(t) d t
$$

and $\sigma_{t}(s)$ is the extinction coefficient which defines the rate of radiation that is scattered or absorbed at thickness $s$ in the medium.

An optically thin medium is, hence, either spatially thin and/or has a small extinction coefficient. Semi-transparent fabric must have a relatively small $\sigma_{t}$ at least in regular places throughout its micro geometry (otherwise it would not be semi-transparent), and since we know that most cloth is spatially quite thin, we can assume that we are working with an optically thin medium when we need to find the light transmitted through some cloth.

Since we would think of the medium around the thin fabric represented by an infinitely thin surface as a slab with the same tangent planes as the original surface, it is entirely reasonable to assume that the medium locally has plane-parallel surfaces.

Having a plane-parallel medium which is of optical thickness $\tau_{1}$, say, we can reformulate (1) in terms of the direction $\pm \boldsymbol{\omega}$ of the radiance and the optical thickness $\tau \in\left[0, \tau_{1}\right]$ below the outward surface location $\boldsymbol{x}$. This gives an expression for outward scattered radiance $L(\tau,+\boldsymbol{\omega})$ and an expression for inward scattered radiance $L(\tau,-\boldsymbol{\omega})$.

Since we are only interested in the emergent radiance on the outward surface, where $\tau=0$, or the inward surface, where $\tau=\tau_{1}$, we can obtain the following through insertion of the optical thicknesses corresponding to the surfaces:

$$
\begin{aligned}
& L(0,+\boldsymbol{\omega})=L\left(\tau_{1},-\boldsymbol{\omega}\right) e^{-\tau_{1} / \mu} \\
& \quad+\int_{0}^{\tau_{1}} \frac{e^{-t / \mu}}{\mu} \int_{\Omega_{4 \pi}} p\left(t, \boldsymbol{\omega}^{\prime}, \boldsymbol{\omega}\right) L\left(t, \boldsymbol{\omega}^{\prime}\right) d \omega^{\prime} d t
\end{aligned}
$$

\footnotetext{
${ }^{1}$ A medium which is not a light source is called a scattering medium
}

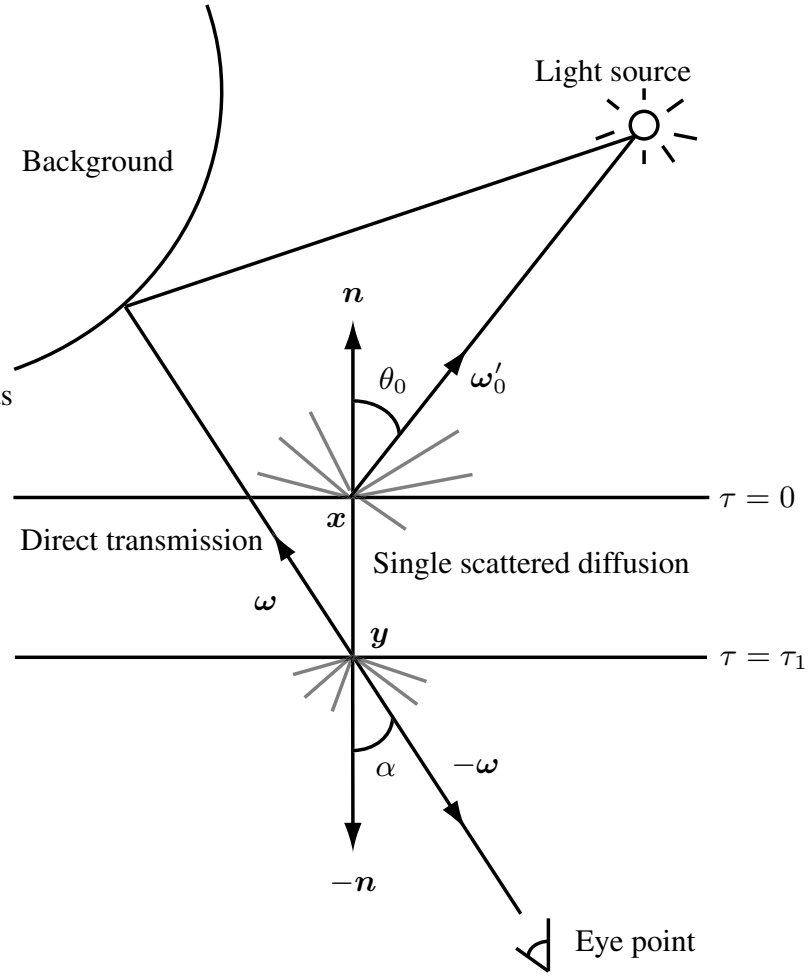

Figure 1: An illustration of the back-lit case where we are looking at the inward surface of a thin plane-parallel medium.

and

$$
\begin{aligned}
& L\left(\tau_{1},-\boldsymbol{\omega}\right)=L(0, \boldsymbol{\omega}) e^{-\tau_{1} / \mu} \\
& \quad+\int_{0}^{\tau_{1}} \frac{e^{-\left(\tau_{1}-t\right) / \mu}}{\mu} \int_{\Omega_{4 \pi}} p\left(t, \boldsymbol{\omega}^{\prime},-\boldsymbol{\omega}\right) L\left(t, \boldsymbol{\omega}^{\prime}\right) d \omega^{\prime} d t
\end{aligned}
$$

If $\boldsymbol{\omega}$ is the direction towards the eye, we calculate the outward emergent radiance (2). If $-\boldsymbol{\omega}$ is the direction towards the eye, we calculate the inward emergent radiance (3). In this formulation of the volume rendering equation, we have

$$
\mu=\cos \alpha=\boldsymbol{n} \cdot \boldsymbol{\omega},
$$

where $\alpha$ is the angle between the outward normal $\boldsymbol{n}$ and the outward direction $\boldsymbol{\omega}$ such that $0<\mu<1$.

Suppose we look, from the direction $-\boldsymbol{\omega}$, at the location $\boldsymbol{y}$ on the surface of a thin medium. Then the direct transmission terms, that is, the first terms of the emergent radiance calculations (2) and (3), capture the radiance reaching the eye from $\boldsymbol{y}$ after it has reached $\boldsymbol{y}$ in a straight line through the medium from the direction $\boldsymbol{\omega}$. In other words, the direct transmission terms account for the transparency of the medium. See figure 1 .

Most fabrics, such as cotton, tend to be quite isotropic ${ }^{2}$ and therefore we can assume for all optical thicknesses $\tau \in\left[0, \tau_{1}\right]$ that

$$
p\left(\tau, \boldsymbol{\omega}^{\prime}, \boldsymbol{\omega}\right)=\frac{\Lambda}{4 \pi},
$$

which is the phase function for isotropic scattering. Though it is a bit old fashioned, the single scattering albedo $\Lambda=\sigma_{s} / \sigma_{t}$, where $\sigma_{s}$ is the scattering coefficient, is included in the phase function in our treatment.

\footnotetext{
${ }^{2} \mathrm{An}$ isotropic medium corresponds to a diffuse surface.
} 
The second terms of (2) and (3), in the following referred to as the diffusion terms, account for diffusely scattered reflection and transmission, respectively, and are more complex to simulate. Since we are working with thin media, it is reasonable to assume that approximation of a single diffuse scattering suffices.

Due to the law of conservation of energy, several principles of invariance with respect to diffusely reflected and transmitted radiance in a plane-parallel medium of optical thickness $\tau_{1}$ can be derived, see [Chandrasekhar 1960, Chap. VII]. Using the laws of diffuse reflection and transmission for isotropic scattering, these principles of invariance can be reduced to a pair of integral equations from which approximations to the diffusion terms can be found, see [Chandrasekhar 1960, Chap. IX]

For optically thin media, ie. for small values of $\tau_{1}$, a single diffuse scattering of light gives an approximation of the diffusion terms in the following way:

$$
L_{+}^{(1)}(0, \mu)=\frac{\Lambda}{4 \pi} E(0) \frac{\mu_{0}}{\mu+\mu_{0}}\left(1-e^{-\tau_{1}\left(\frac{1}{\mu}+\frac{1}{\mu_{0}}\right)}\right)
$$

and

$$
L_{-}^{(1)}\left(\tau_{1}, \mu\right)=\frac{\Lambda}{4 \pi} E(0) \frac{\mu_{0}}{\mu-\mu_{0}}\left(e^{-\tau_{1} / \mu}-e^{-\tau_{1} / \mu_{0}}\right),
$$

where $E(0)$ is the irradiance incident at the outward surface location $\boldsymbol{x}$ corresponding to $\tau=0$, and $\mu_{0}=\cos \theta_{0}=\boldsymbol{\omega}_{0}^{\prime} \cdot \boldsymbol{n}$ is cosines of the angle between the normal at $\boldsymbol{x}$ and the direction $\boldsymbol{\omega}_{0}^{\prime}$ towards the light source. Single scattering is also illustrated in figure 1 . Note that the diffusion terms depend on $\mu$ not the direction of the emergent radiance $\pm \boldsymbol{\omega}$ itself. The reason for this is the assumption of isotropic scattering.

Equations (4) and (5) are very similar to the equations derived in [Blinn 1982]. Except for the detail that Blinn has $\mu_{0}<0$ while we always have $0<\mu_{0} \leq 1$, and he calculates brightness (radiant flux per projected solid angle) whereas we calculate radiance. To accommodate more sophisticated materials, Blinn also replaces the phase function for isotropic scattering $p\left(t, \boldsymbol{\omega}^{\prime}, \boldsymbol{\omega}\right)=\Lambda /(4 \pi)$ with $p\left(t, \boldsymbol{\omega}^{\prime}, \boldsymbol{\omega}\right)=\Lambda p^{\prime}\left(\boldsymbol{\omega} \cdot \boldsymbol{\omega}_{0}^{\prime}\right)$, where $p^{\prime}$ is a normalized phase function. This is of course also possible in the derivation presented here.

A few simple calculations show that the irradiance incident on the surfaces visible from an isotropic point light source, emitting a constant flux $\boldsymbol{\Phi}_{s}$ in all directions, is

$$
E(\boldsymbol{x})=\frac{\boldsymbol{\Phi}_{s}}{4 \pi} \frac{\cos \theta_{0}}{r^{2}}=\frac{\boldsymbol{\Phi}_{s} \mu_{0}}{4 \pi r^{2}},
$$

where $r^{2}$ is the distance between the surface location $\boldsymbol{x}$ and the position of the point light source.

Replacing the diffusion terms in (2) and (3) with the approximations given in (4) and (5), respectively, we get the following rendering equations for optically thin, locally plane-parallel mediums represented by surfaces:

$$
L(0,+\boldsymbol{\omega})=L\left(\tau_{1},-\boldsymbol{\omega}\right) e^{-\tau_{1} / \mu}+L_{+}^{(1)}(0, \mu)
$$

and

$$
L\left(\tau_{1},-\boldsymbol{\omega}\right)=L(0, \boldsymbol{\omega}) e^{-\tau_{1} / \mu}+L_{-}^{(1)}\left(\tau_{1}, \mu\right)
$$

where the expression (6) for irradiance resulting from a point light source could be inserted in the calculation of the diffusion terms, or the irradiance could be calculated in an alternative way, eg. using photon mapping.

Equations (7) and (8) incorporate both direct transmission through the medium and an approximate contribution of light that has suffered from a single scattering in the medium. For $\tau_{1} \leq 0.2$, the diffusion approximation differs less than 1 per cent from the correct value [Chandrasekhar 1960, p. 205]. Equation (7) is evaluated when the surface is front-lit and (8) is evaluated when the surface is back-lit.

\section{Rendering Thin Objects}

In this section, we will give an example of the visual effects resulting from application of the theory described in the previous section. As a simple case study, we will render a waving flag against a background painting. To illustrate the different lighting effects, we will have a point light source circulating the flag.

As the method prescribes, the flag is represented by a surface and the normals are always turned outwards such that $\mu_{0}=\cos \theta_{0}>0$. Equations (6), (7), and (8) are evaluated in a fragment program. With a slight loss of quality, they can as well be evaluated for each vertex. This could happen in a vertex program or on the CPU.

The direct transmission term represents light which has moved in a straight line through the medium. This term can, therefore, be rendered using alpha blending with

$$
\alpha_{\text {source }}=e^{-\tau_{1} / \mu}
$$

and a blending function such that

$$
L_{\text {blended }}=L_{\text {destination }} \alpha_{\text {source }}+L_{\text {source }},
$$

where $L_{\text {blended }}$ is the final pixel value, $L_{\text {destination }}$ is the previous pixel value, and $L_{\text {source }}$ is the color calculated in the fragment program. The alpha blending factor $\alpha_{\text {source }}$ should also be calculated in the fragment program.

Clearly (9) captures either (7) or (8) depending on whether we let $L_{\text {source }}$ be calculated in the fragment program as $L_{+}^{(1)}(0, \mu)$ or $L_{-}^{(1)}\left(\tau_{1}, \mu\right)$. Of course this depends merely on whether $\boldsymbol{\omega}$ or $-\boldsymbol{\omega}$ is the direction towards the eye.

Observe that the alpha blending value we use is not constant. It depends on $\mu=\boldsymbol{n} \cdot \boldsymbol{\omega}$ which changes with cosines of the angle between the outward normal $\boldsymbol{n}$ and the outward direction $\boldsymbol{\omega}$. On the other hand it is interesting to note that the direct transmission used for alpha blending actually is the same both for back-lit and front-lit surfaces. The reason why back-lit surfaces often appear to be more transparent is that they are usually darker why the direct transmission term is more readily perceptible.

The model we present indeed gives improved results as compared to evaluation of a BRDF combined with a constant for alpha blending. Traditionally there are two ways to deal with surface representations of thin objects. Either back-lit surfaces are rendered as if they were front-lit or they are shadowed and completely black except for the constant alpha-blending. In figure 2, renderings of the last case are compared to corresponding renderings using the presented method.

The frames rendered in the presented example are of resolution $512 \times 512$. On a $2.13 \mathrm{GHz}$ Pentium M laptop with a Quadro FX Go 1400 graphics card, the method runs approximately in $300 \mathrm{fps}$ (frames per second) when implemented in a fragment program. In a vertex program it runs in $480 \mathrm{fps}$. If calculations are done on the CPU, we obtain $190 \mathrm{fps}$. On an older $1 \mathrm{GHz}$ Pentium 3 with a GeForce4 MX 440, the method runs in $330 \mathrm{fps}$ using a vertex program and in $150 \mathrm{fps}$ using the CPU. This shows that the method has no problems running in real-time.

The background painting in our case study is a watercolor painting picturing a view at the Faroe Islands. The chosen flag is, therefore, the flag belonging to the Faroe Islands.

\section{Discussion and Conclusion}

Having surface representations of thin semi-transparent objects such as garments, it has hitherto been difficult to account realistically for the light transmitted directly through, and the light diffusely scattered by, the thin medium which the surface represents. In particular, it has been difficult to account realistically for the 


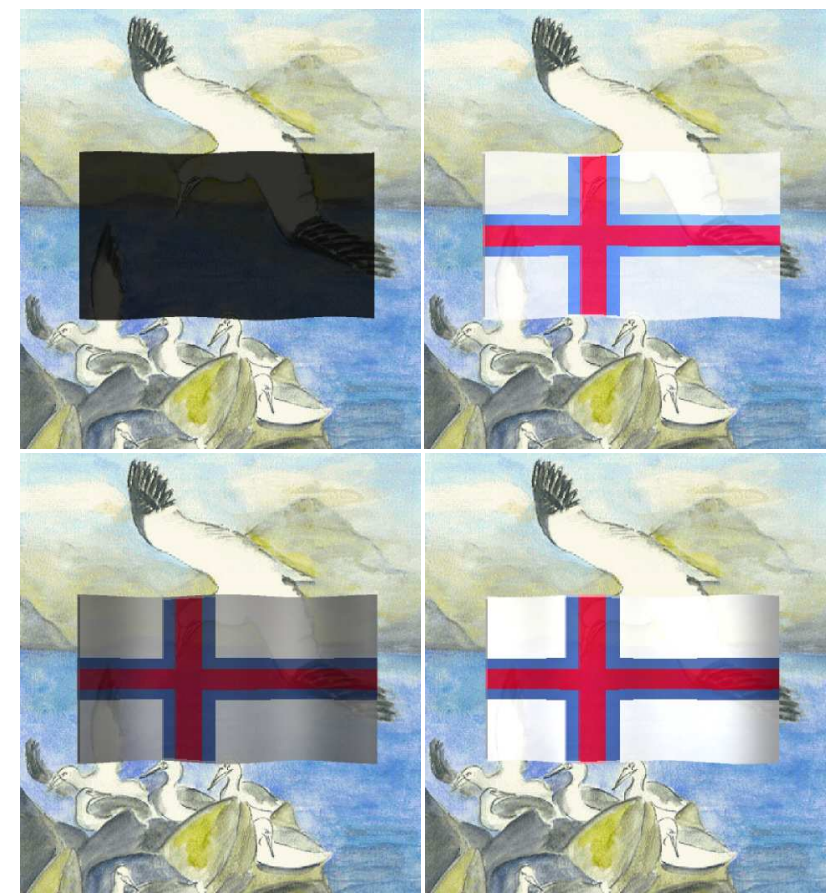

Figure 2: Renderings of a flag against a background painting. The top row pictures uses the traditional rendering equation and a constant alpha-blending with $\alpha_{\text {source }}=0.2$. The bottom row uses the rendering scheme presented in this paper with $\Lambda=1$ and $\tau_{1}=2$. The flag is back-lit in the left column and front-lit in the right column.

nonconstant transparency of thin cloth as well as the obviously different appearance of back-lit cloth as opposed to front-lit cloth. In this paper, a simple rendering scheme capturing such visual effects has been presented.

The described rendering model is similar to the one presented in [Blinn 1982] for rendering of media with low density, eg. clouds and smoke. Since the same rendering method can be derived from a different set of assumptions, we have demonstrated that it is also applicable to surface representations of thin objects. In other words, our observation is that a well known rendering technique can be used for realistic real-time rendering of a different category of objects than it was originally intended for. In particular, we have demonstrated that Blinn's method gives improved realism in cloth rendering though it was intended for rendering of participating media such as clouds.

The only parameters which the model depends on are the optical thickness $\tau_{1}$ and the single scattering albedo $\Lambda$ of the object to be rendered. A face function depending on the angle between the direction towards the eye and the direction towards the light source can be introduced to approximate scattering from materials that are not completely isotropic.

For future experimentations, the model could be combined with existing surface models for cloth rendering. For example it could be interesting to associate the optical thickness of a fabric with a bump map accounting for micro-geometric details. In that case, the thickness would vary across the surface according to the bump map. This may violate the assumption of plane-parallel media on a microscopic level, but the assumption is still preserved on a macroscopic level why the approach may be acceptable.

In the real-time implementation we present, the non-constant alpha blending can account for multiple layers of cloth, but the diffuse single scattering is calculated locally and cannot account for scat- tering of light through multiple layers. An interesting direction for future works could therefore be to incorporate the rendering model in a photon mapping solution. The photon tracing would be able to account for diffuse transmission through multiple surfaces given a proper BSDF, while the presented method should be used in the radiance estimate, since the traditional radiance estimate is unable to account for the emergent radiance on the backside of an object represented solely by a surface.

\section{Acknowledgement}

Thanks to Felicia Frisvad for letting us use a scan of her watercolor painting as the background in our case study.

\section{References}

Adabala, N., Magnenat-Thalmann, N., and Fei, G. 2003. Real-time rendering of woven clothes. In Proc. of the ACM Symposium on Virtual Reality Software and Technology, 41-47.

AdABALA, N. 2004. Rendering of skin and clothes. In Handbook of Virtual Humans, N. Magnenat-Thalmann and D. Thalmann, Eds. John Wiley \& Sons, ch. 15, 353-372.

BLINN, J. F. 1982. Light reflection functions for simulation of clouds and dusty surfaces. Computer Graphics (SIGGRAPH '82 Proceedings) 16, 3 (July), 21-29.

Chandrasekhar, S. 1960. Radiative Transfer. Dover Publications, Inc., New York. Unabridged and slightly revised edition of the work first published in 1950.

Chen, Y., Lin, S., Zhong, H., Xu, Y.-Q., Guo, B., And SHUM, H.-Y. 2003. Realistic rendering and animation of knitwear. IEEE Transacions on Visualization and Computer Graphics 9, 1 (January), 43-55.

Daubert, K., AND SeIDEL, H.-P. 2002. Hardware-based volumetric knit-wear. Computer Graphics Forum (Proc. of Eurographics 2002) 21, 3, 575-584.

Daubert, K., Lensch, H. P. A., Heidrich, W., AND Seidel, H.-P. 2001. Efficient cloth modeling and rendering. In Proc. of the Twelfth Eurographics Workshop on Rendering Techniques, Springer, S. J. Gortler and K. Myszkowski, Eds., 63-70.

Kajiya, J. T., ANd Von Herzen, B. P. 1984. Ray tracing volume densities. Computer Graphics (SIGGRAPH '84 Proceedings) 18, 3 (July), 165-174.

MAX, N. 1995. Optical models for direct volume rendering. IEEE Transactions on Visualization and Computer Graphics 1, 2 (June), 99-108.

Messnier, M., Eberhardt, B., And Strasser, W. 2000. A volumetric appearance model. In Cloth Modeling and Animation, D. H. House and D. E. Breen, Eds. A K Peters, Natick, Massachusetts, ch. 11, 269-285.

Rushmeier, H. E., And Torrance, K. E. 1987. The zonal method for calculating light intensities in the presence of a participating medium. Computer Graphics (SIGGRAPH '87 Proceedings) 21, 4 (July), 293-302.

Volevich, V. L., Kopylov, E. A., Khodulev, A. B., AND KARPENKO, O. A. 1997. An approach to cloth synthesis and visualization. In Proc. of GraphiCon '97, the Seventh Internation Conference on Computer Graphics and Visualization, S. Klimenko, Ed. 